\title{
Spot urine collection: A valid alternative to total urine collection for metabolomic studies in dairy cattle
}

\author{
H. Boudra, ${ }^{1 *}$ P. Noziere, ${ }^{1}$ ○ G. Cantalapiedra-Hijar, ${ }^{1} \odot$ M. Traikia, ${ }^{2}$ J-F. Martin, ${ }^{3}$ M. Petera, ${ }^{2} \odot$ M. Lagree, ${ }^{2}$ \\ M. Doreau, ${ }^{1}$ and D. P. Morgavi ${ }^{1 *}$ (1) \\ ${ }^{1}$ Université Clermont Auvergne, INRAE, VetAgro Sup, UMR Herbivores, F-63122 Saint-Genes-Champanelle, France \\ ${ }^{2}$ Université Clermont Auvergne, INRAE, UNH, Plateforme d'Exploration du Métabolisme, MetaboHUB Clermont, F-63000 Clermont-Ferrand, \\ France \\ ${ }^{3}$ INRAE, Toxalim, MetaboHub-Axiom, Metabo-Hub-ANR611-INBS-0010
}

\section{ABSTRACT}

Urine is a highly suitable biological matrix for metabolomics studies. Total collection for 24-h periods is the gold standard as it ensures the presence of all metabolites excreted throughout the day. However, in animal studies, it presents limitations related to animal welfare and also due to alterations of the metabolome originating from the use of acid for preventing microbial growth or microbial contamination. In this study, we investigated whether spot urine collection is a practical alternative to total collection for metabolomic studies in lactating cows. For this purpose, we collected urine samples from 4 lactating Holstein cows fed 4 diets in a $4 \times 4$ Latin square design. Urine was collected for $24 \mathrm{~h}$ using a collecting device (i.e., total collection) or collected once per day $4 \mathrm{~h}$ after the morning feeding (i.e., spot urine collection). Dietary treatments differed by the amount of nitrogen content (high vs. low) and by the nature of the energy (starch vs. fiber). Urine metabolome was analyzed by 2 untargeted complementary methods, nuclear magnetic resonance and hydrophilicinteraction liquid chromatography (HILIC) coupled to a time-of-flight mass spectrometer, and by 1 targeted method, HILIC-tandem mass spectrometry. Although sampling technique had an effect on the abundance of metabolites detected, spot urine samples were equally capable of showing differences in urine metabolome than samples from total collection. When considering nitrogen levels in the diet, the robustness and precision for discriminating high- and low-nitrogen diets was equally achieved with both sampling techniques. A total of 22 discriminant metabolites associated with the $\mathrm{N}$ level of diets were identified from untargeted HILIC

Received May 26, 2021.

Accepted September 13, 2021.

*Corresponding authors: abdelhamid.boudra@inrae.fr and diego. morgavi@inrae.fr coupled to a time-of-flight mass spectrometer $(\mathrm{n}=9)$ and nuclear magnetic resonance $(\mathrm{n}=11)$, and 2 from targeted HILIC-tandem mass spectrometry. Alternatively, starch or fiber in the diet induced less changes in the metabolome that were not clearly discriminated independently of the sampling technique. We concluded that spot urine collection can successfully reveal differences in the urine metabolome elicited by dietary $\mathrm{N}$ levels and be used as a substitute of total urinary 24-h collection for metabolomic studies.

Key words: urine, lactating dairy cow, nitrogen diet, metabolomics, spot collection

\section{INTRODUCTION}

Metabolomics is a powerful tool for studying metabolic changes in mammals (Sun et al., 2015; ContrerasJodar et al., 2019). Urine is a suitable matrix when compared with other biofluids such as plasma, as it can be obtained noninvasively. In addition, unlike plasma and milk, urine samples do not require extraction with organic solvents that may affect the metabolome, making it the matrix of choice in many studies (Dunn et al., 2011; Vuckovic, 2012).

Urine is collected in 2 ways, total collection (TC) performed over 1 or several pooled 24 -h periods, and spot collection (SC) performed at different times and frequencies throughout the day. Total collection is considered the gold standard as it provides a representative sample that can be used for quantifying the daily metabolite excretion from individual animals, whereas $\mathrm{SC}$ is less precise as it is based on models and indirect measures to estimate daily urine output. Indeed, total urinary excretion of specific metabolites is currently used for estimating key physiological processes in ruminants such as the rumen microbial protein flow to the duodenum (i.e., from urinary purine derivatives excretion; Chen and Gomes, 1992) or the myofibrillar protein degradation rate (i.e., from 3-methyl-histidine urinary excretion; Harris and Milne, 1981). However, 
in research with animals, and ruminants in particular, there are many drawbacks linked to this type of collection. Total urine collection requires the animals be housed in tiestalls, reducing the number of individuals that can be used in experiments (Wilson et al., 2019). Also, tethering and tiestall systems pose additional welfare issues for cows (Nalon and Stevenson, 2019). Additionally, it is not possible to perform TC under grazing conditions. It is also noted that TC is complicated as it requires the use of harnesses to hold collection devices or, in the case of females, invasive indwelling catheters. Moreover, TC using noninvasive devices is associated with a higher risk of macroscopically undetected fecal contamination that can modify the urinary metabolome by adding fecal metabolites or by degrading already-present metabolites through the action of contaminating microbes (Patra and Aschenbach, 2018). The negative effect of fecal contamination may be amplified by high temperature and exposure to light (Boomsma et al., 1993; Fernández-Peralbo and Luque de Castro, 2012; Kirwan et al., 2018). Addition of chemical preservatives such as sulfuric acid reduces the risk of microbial growth but can also alter the urine metabolome. Sulfuric acid does react with some compounds, such as alcohols, steroids, sugars, or proteins, causing degradation and modifications of metabolites into several end products. Metabolite degradation, adducts formation, and ion pairing created by the strong acid undermines sensitivity of MS (Chowdhury et al., 1990; Furey et al., 2013), diminishing the discovery potential of untargeted metabolomics. In contrast, SC is an easier and rapid way to collect urine samples that reduces contamination and avoids the use of preservatives. In addition, SC aligns with current welfare recommendation in dairy production (Nalon and Stevenson, 2019). We hypothesized that SC could provide similar insight as TC on the urine metabolome of lactating cows. The objective of this study was to compare the potential of SC and TC to discriminate differences in the metabolome. To have a more complete image of the urine metabolome and a more robust comparison between sampling techniques, we used untargeted and targeted metabolomic methods. We used lactating dairy cows that were fed differing amounts of nitrogen and different types of energy sources. A secondary objective of this work was to identify urinary metabolites that reflected differences in diet composition.

\section{MATERIALS AND METHODS}

\section{Animals, Diets, and Sample Preparation}

The experiment was conducted at the animal experimental facilities of the HerbiPole at Marcenat, INRAE, in 2018. Low Mountain Ruminant Farming Systems Facility, DOI 10.15454/1.5572318050509348E12. Procedures with animals were conducted in accordance with the guidelines for animal research of the French Ministry of Agriculture and applicable European guidelines and regulations for experimentation with animals (https://eur-lex.europa.eu/legal-content/EN/TXT/ $\mathrm{PDF} /$ ?uri $=$ CELEX:32010L0063\&from $=\mathrm{EN})$.

The experimental design was previously described (Fanchone et al., 2013). Briefly, 4 multiparous (parity $=2.5 \pm 0.6$; mean $\pm \mathrm{SD}$ ) lactating Holstein dairy cows were fed diets containing $11 \% \mathrm{CP}$ (low N) or $14 \% \mathrm{CP}$ (high N) with high $(30 \%)$ or low $(15 \%)$ starch. Cows were $4.7 \pm 0.9 \mathrm{yr}$ old, weighed $662 \pm 62 \mathrm{~kg}$, and were $71 \pm 10$ DIM at the beginning of the study. Diets were assigned to the cows during 4 successive periods in a $4 \times 4$ Latin square design. Each experimental period lasted $28 \mathrm{~d}$ and consisted of $23 \mathrm{~d}$ of adaptation to the diet and $5 \mathrm{~d}$ of measurements. A transition period of 3 d was applied between each experimental period. Animals were fed twice daily at 0900 and $1700 \mathrm{~h}$. Diets had similar net energy values and proportion of forage to favor similar DM intake and avoid confounding effects on digestibility between diets due to level of intake. Diet composition is shown in Supplemental Table S1 (https://data.inrae.fr/dataset.xhtml? persistentId=doi: 10.15454/FFFRL2, Morgavi, 2021). Daily DMI and milk production throughout the study was, respectively, $20.2 \pm 0.8 \mathrm{~kg}$ of DM and $23.7 \pm 2.3 \mathrm{~kg}$ of milk per cow.

Urine samples were daily collected during the last week of treatment over 5 consecutive days. They were collected separately in 2 different ways. Total urine samples were collected during 24-h periods using caps attached to the vulva and connected to a $30-\mathrm{L}$ collection carboy via a flexible tube. The carboy contained $500 \mathrm{~mL}$ of $10 \%$ (vol/vol) sulfuric acid as preservative. Every day before the morning feeding, a 100-mL aliquot was taken and the carboy was replaced with a clean one. Spot urine samples were taken between 4 and $6 \mathrm{~h}$ after the morning feeding (1300 to $1500 \mathrm{~h}$ ). The perineal region was wiped with a clean paper towel to remove fecal residues, and urination was stimulated by massaging the vulva. After urination started, the first milliliters were discarded and urine was collected into a clean beaker without the use of any preservative. Out of 80 samples planned for each sampling technique, we collected 71 for TC (9 samples excluded due to fecal contamination) and 74 for SC (6 samples, obtained from cows in heat, were discarded as urine did not have the usual aspect). Samples from both types of collection were immediately centrifuged at $10,000 \times g$ at $4^{\circ} \mathrm{C}$ for $10 \mathrm{~min}$, filtered through $0.45-\mu \mathrm{m}$ single-use syringe filter (Sartorius) to remove debris, aliquoted, and stored at $-80^{\circ} \mathrm{C}$ until analysis. Storage time was 17 mo. 


\section{Metabolite Analysis}

For a wide coverage of the metabolome and to deal with the highly diverse range of metabolites present in urine, different analytical techniques for both untargeted and targeted analysis are used (Schrimpe-Rutledge et al., 2016). We used 3 analytical platforms as follows: 2 complementary untargeted nuclear magnetic resonance (NMR) and hydrophilic-interaction liquid chromatography (HILIC) coupled to a time-of-flight mass spectrometer (HILIC-ToF/MS), and by 1 targeted method HILIC-tandem mass spectrometry (HILICMS/MS). For untargeted methods, daily collected urine samples ( $\mathrm{n}=71$ for $\mathrm{TC}, \mathrm{n}=74$ for $\mathrm{SC}$ ) were analyzed. For the targeted method, we analyzed pooled samples per animal that were obtained by combining an equal volume of urine collected in each period and for each sampling technique $(n=16 \times 2)$.

Metabolite profiling using a discovery-based, untargeted approach of global detection of all metabolites present in urine was performed on a Metabolic Profiler system that combined 2 techniques of detection, MS and NMR. The Metabolic Profiler consisted of an AVANCE III $500 \mathrm{MHz}$ NMR spectrometer equipped with a 3-mm FISEI z-gradient $\left({ }^{1} \mathrm{H}^{13} \mathrm{C}\right)$ probe and a $60-\mu \mathrm{L}$ flow cell (Bruker Biospin $\mathrm{GmbH}$ ) and a liquid chromatography coupled (LC) apparatus (Agilent 1100 HPLC system) coupled to a MicrOTOF (time-of-flight) mass spectrometer (Bruker Daltonics).

HILIC-ToF/MS Analysis. Urine samples (100 $\mu \mathrm{L})$ were thawed at room temperature, and a $100-\mu \mathrm{L}$ aliquot was transferred into a clean Eppendorf tube and mixed with $300 \mu \mathrm{L}$ of acetonitrile. The samples were vortex-mixed and centrifuged at $10,000 \times g$ for 10 min. Then, supernatants were transferred into an autosampler, and an aliquot of $10 \mu \mathrm{L}$ was injected into the LC-MS system. To ensure performance of analytical instrumentation and that the data were of comparable high quality within and between series, quality control samples (QC) were inserted in duplicate every 5 urine samples. Two types of QC were prepared by pooling samples from SC and TC collections. Each pooled QC was obtained by mixing equal volume of urine from samples of all cows over the 4 experimental periods. These QC samples were processed as above. Separation was performed on a $150 \times 2 \mathrm{~mm}$ i.d. HILIC column (Phenomenex) fitted with a same type $2 \times$ $2 \mathrm{~mm}$ guard column, and using a water/acetonitrile (both containing $5 \mathrm{mM}$ ammonium formate, $\mathrm{pH}$ 6.8) gradient at a flow rate of $0.3 \mathrm{~mL} / \mathrm{min}$. The gradient was started at $5 \%$ water, held for $2 \mathrm{~min}$, and increased to $50 \%$ in $12 \mathrm{~min}$; $50 \%$ water was maintained for 2 min before the gradient was returned to initial conditions and maintained for $7 \mathrm{~min}$ for re-equilibrating the column prior the next injection. The MS system was operated in positive ionization mode with a scan range of 50 to $800 \mathrm{~m} / z$. The capillary was set to $-4.5 \mathrm{kV}$, the nebulizer was operated at 2 bars, and the dry gas was set to $8 \mathrm{~L} / \mathrm{min}$ at a temperature of $200^{\circ} \mathrm{C}$. The capillary exit was set to $90 \mathrm{~V}$ with skimmer 1 set to $30 \mathrm{~V}$. The time-of-flight spectrometer was calibrated by using lithium formate (ions at $m / z 90$ and 800). The MS source was regularly cleaned after 100 injections. For accurate mass acquisition, a formate-acetate solution was infused during the run at a flow rate $100 \mu \mathrm{L} / \mathrm{min}$ monitoring for positive ion mode.

1-Dimensional NMR Analysis. Urine samples were centrifuged at $10,000 \times g$ for $10 \mathrm{~min}$ at $4^{\circ} \mathrm{C}$. To $200 \mu \mathrm{L}$ of supernatant of each sample, the robot of the Metabolic Profiler facility added $30 \mu \mathrm{L}$ of a phosphate buffer $\left(\mathrm{KH}_{2} \mathrm{PO}_{4}\right.$ and $\mathrm{K}_{2} \mathrm{HPO}_{4}$, Fluka; $\mathrm{pH}$ 7.06) in Deuterium oxide (99.96\% minimum, Eurisotop), containing $4.6 \mathrm{~m} M \quad 3$-(trimethylsilyl) propionic-2,2,3,3-tetra-d4 acid sodium salt (TSP-d4; 98\% deuterated, Sigma-Aldrich), and $6.1 \mathrm{~m} M$ sodium azide (NaN3, 99.99\% minimum, Sigma-Aldrich). The ${ }^{1} \mathrm{H}$ NMR experiments were carried out on a Bruker Metabolic Profiler equipped with an AVANCE III $500 \mathrm{MHz}$ NMR spectrometer using a 3-mm FISEI z-gradient $\left({ }^{1} \mathrm{H}^{-13} \mathrm{C}\right)$ probe with a 60 $\mu \mathrm{L}$ flow cell (Bruker Biospin). Cell probe was filled with sample using Gilson 215 robot incorporated into BEST (Bruker Efficient Sample Transfer) system. A standard monodimensional nuclear Overhauser effect spectroscopy sequence (noesygppr1d with water presaturation and gradients) was used with low power irradiation of the water resonance during the recycle delay of $4 \mathrm{~s}$ and the mixing time of $10 \mathrm{~ms} ; 128$ scans were collected with a $90^{\circ}$ impulsion time of $9.3 \mu \mathrm{s}$, an acquisition time of $3.2 \mathrm{~s}$, and a spectral window of $10,000 \mathrm{~Hz}$; and $64 \mathrm{~K}$ data points zero-filled to $128 \mathrm{~K}$ before Fourier transformation with $0.3 \mathrm{~Hz}$ line broadening. All NMR spectra were recorded at $300 \mathrm{~K}$. The robot maintained samples in well plates at $4^{\circ} \mathrm{C}$ during analyses.

HILIC-MS/MS Analysis. We used a targeted approach to do the absolute quantification of metabolites related to nitrogen status. Urine samples from the 2 sampling techniques were analyzed as previously described (Boudra et al., 2012). Briefly, urine samples collected from each cow over 5 consecutive days were pooled. The mixture $(100 \mu \mathrm{L})$ was diluted with acetonitrile, vortex-mixed and centrifuged, and $10 \mu \mathrm{L}$ were injected into the LC-MS/MS system.

\section{Data Processing}

The MS data were converted to NetCDF format using 3.4 Datanalysis software (Bruker Biospin), and MS data from untargeted approach were processed using 
the open-source web-based interface Galaxy instance Workflow4Metabolomics running under R3.5.2 (Giacomoni et al., 2015). Further pre-processing steps were also applied to remove noise and unwanted variation, including filtrations and normalization. The MS data were first filtered on retention time, and signals outside the range (lower than $0.4 \mathrm{~min}$ or higher than $22 \mathrm{~min}$ ) were considered as noise and removed. Background noise was also removed by removing masses found in blank samples (solvents). Because we had 2 types of QC (1 per type of collection), MS data of each collection were then normalized within batch using a linear regression model (van der Kloet et al., 2009). A data matrix containing mass and retention time with associated signal intensities for all detected peaks was generated. An additional filtering test was used to select variables based on univariate analysis (Mann-Whitney-Wilcoxon test on $\mathrm{N}$ levels); the ions with $P<0.3$ were retained for further multivariate analysis. After denoising and filtering tests, the number of features was reduced from 4,662 to 2,177 (47\%).

The NMR data processing was performed in 2 steps. First, zero- and first-order phase correction was achieved manually on all spectra. Baseline correction was performed using a third-order polynomial. Chemical shifts in all spectra were referenced to the TSP$\mathrm{d} 4$ signal at chemical shift $(\delta=0 \mathrm{ppm})$. Spectra were treated with TopSpin version 2.1. Then, bucketing was performed using AMIX 3.9.10. The NMR spectra were subdivided in narrow spectral regions (0.02-ppm wide simple rectangular buckets). The spectral region from 10.50 to $0.50 \mathrm{ppm}$ (spot urine) and 9.14 to $0.65 \mathrm{ppm}$ (total urine) were used for bucketing. Spectral regions that either did not contain any resonances for SC samples (10.00-9.36 ppm and 0.65-0.50 ppm) or contained the residual water resonance (5.50-4.72 ppm for all samples; for TC samples: 5.60-4.72 ppm) were excluded from the bucketing procedure. Due to the chemical exchange with water, the urea peak is influenced by water pre-saturation, which requires the elimination of this peak (Beckonert et al., 2007). Unfortunately, this excluded the region containing proton chemical shifts of allantoin and saccharides anomeric protons. As for MS data, a table was generated containing the buckets, their chemical shift, and the intensity.

\section{Metabolite Annotation}

Two-dimensional (2-D) NMR data were acquired on a Bruker AVANCE I spectrometer operating at 500 $\mathrm{MHz}$ and equipped with a 5 -mm inverse-triple tuned (TXI) $1 \mathrm{H} / 13 \mathrm{C} / 15 \mathrm{~N}$ with z-gradient coil probe (Bruker Biospin Wissenbourg). For 1-dimensional (1-D) ${ }^{1} \mathrm{H}-$ spectra, a standard 1-D spectroscopy sequence (zgpr) was used with low power irradiation of the water resonance during the recycle delay of $5 \mathrm{~s}$. Then, 128 scans were collected with a $90^{\circ}$ impulsion time of $10 \mu \mathrm{s}$, an acquisition time of $3.2 \mathrm{~s}$, and a spectral window of $10 . \mathrm{k}$ $\mathrm{Hz}$, and $64 \mathrm{~K}$ data points were zero-filled to $128 \mathrm{~K}$ before Fourier transformation with $0.3-\mathrm{Hz}$ line broadening. Two-dimensional homonuclear $(1 \mathrm{H}-1 \mathrm{H}$ COSY, $1 \mathrm{H}-$ $1 \mathrm{H}$ TOCSY, 1H-1H Jres) and heteronuclear $(1 \mathrm{H}-13 \mathrm{C}$ HSQC and HMBC) experiments were performed with quadrature phase detection in dimensions, using stateTPPI (time proportional phase incrementation) or QF (quadrature fixed) detection mode in the indirect experiment. For each of 512 increments in the indirect dimension, $2 \mathrm{~K}$ data points were collected, and 16 or 32 transients were accumulated in the direct dimension. The 13C decoupling (GARP) was performed during acquisition time for heteronuclear experiments. A $\pi / 2$ shifted square sine-bell function was applied in the both dimensions before Fourier transformation. All spectra were recorded at $300 \mathrm{~K}$ and treated with TopSpin version 3.1 software.

For untargeted MS data, mass spectra were first manually annotated for each discriminant ion to identify the parent ion. The redundant $m / z$ peaks corresponding to different ions (fragments and adducts) were used to help confirm the molecular ion, and then removed. For identification, an in-house database containing more than 1,000 metabolites analyzed under the same conditions was first queried based on both retention time and mass obtained from the time-of-flight-MS detector. This identification was based on both their retention time and mass. For the rest of unidentified metabolites, 4 online chemical databases were queried by accurate mass to search for hypotheses of chemical structures as follows: the Human Metabolome Database (HMDB, www.hmdb.ca), Kyoto Encyclopedia of Genes and Genomes (KEGG, http://www.genome.jp/kegg/ ), Metlin (http://metlin.scripps.edu/), and the Bovine Metabolome Database (http://www.rumendb.ca/). To obtain exact masses, pooled urine samples were analyzed using a high resolution Thermo Scientific LTQ Orbitrap Velos hybrid mass spectrometer (Thermo) using the same $\mathrm{LC}$ conditions as the $\mathrm{LC}-\mathrm{MS}$ acquisitions.

The NMR metabolites identification based on the 1-D and 2-D NMR spectra were performed by querying an in-house database, public databases HMDB (Wishart et al., 2018), Biological Magnetic Resonance Data Bank (Ulrich et al., 2008), and relevant publications (Fan, 1996; Beckonert et al., 2007; Bertram et al., 2011).

Eleven urinary metabolites that contributed to the separation of diets differing in $\mathrm{N}$ level were identified. They were confirmed by NMR 2-D in urine spiked by pure standard. The 2-D experiments were performed on pooled urine samples that were prepared as follows. In 
the initial step, 5 QC samples (see in the HILIC-MS experiments section the description of their preparation) were pooled together in an Eppendorf and centrifuged at $10,000 \times g$ at $4^{\circ} \mathrm{C}$ for 30 min on a tabletop centrifuge (Sigma 112). The supernatant was transferred into a $15-\mathrm{mL}$ polypropylene tube and lyophilized. The lyophilized sample was then dissolved into $1.4 \mathrm{~mL}$ of a $39 \mathrm{mM}$ potassium phosphate buffer $\left(\mathrm{pD}^{*} \sim 7.1 ; \mathrm{pH}\right.$ measured in deuterium oxide solvent) prepared in $100 \%$ Deuterium oxide containing $0.6 \mathrm{mM} \mathrm{NaN}$ and $0.8 \mathrm{~m} M$ TSP-d4. Dilution of this pooled QC sample was necessary to facilitate tuning and matching of the NMR probe owing to the high salt content of the sample.

\section{Calculations and Statistical Analyses}

Milk N-use efficiency was calculated as the average daily milk $\mathrm{N}$ secretion divided by the average daily $\mathrm{N}$ intake over 6 consecutive days. This overall milk N-use efficiency was then partitioned into ruminal, digestive, and metabolic efficiencies as previously described (Cantalapiedra-Hijar et al., 2018). Briefly, rumen N-use efficiency was calculated as the microbial protein synthesis divided by rumen degradable protein. Digestive N-use efficiency was computed as the metabolizable protein supply divided by the dietary CP intake, whereas metabolic N-use efficiency was calculated as the milk N secretion divided by the metabolizable protein supply. All variables used in these calculations were measured and not estimated (Fanchone et al., 2013).

Combined univariate and multivariate analyses were performed to compare urine metabolites in both sampling techniques. Before statistical analyses, both NMR and MS data were processed and subsequently normalized to the total intensity to avoid bias due to normal variations in concentration between cows and due to the collection method. Data were then subjected to univariate scaling and analyzed using SIMCA-P software (Umetrics, v. 13.01). Unsupervised principal component analysis (PCA) was first used to visualize trends in metabolite profiles between TC and SC, followed by supervised orthogonal partial least squares (OPLS)-discriminant analysis (DA) to discriminate N diets. Validation of the OPLS models were performed by considering samples from each day collection independently. Discriminant variables were selected according to the variable's importance in projection (VIP; VIP $\geq 1.0$ ). These discriminant variables were then subjected to annotation (Supplemental Methods; https: / /data.inrae.fr/dataset.xhtml? persistentId=doi: 10.15454/FFFRL2, Morgavi, 2021). The characteristics of the OPLS models were assessed by the following: proportion of the dependent variables that is explained by the model $\left(\mathrm{R}^{2} \mathrm{X}\right)$, cumulative predictive ability parameter $\left(Q^{2}\right)$, and the number of VIP $\geq 1$, indicating the contribution of variables to the discrimination between $\mathrm{N}$ diets. All OPLS models were tested for overfitting with a 200-time permutation analysis [see Worley and Powers $(2013,2016)$ for further details and examples of the statistical analyses].

Further univariate analyses (2-way ANOVA, cow, and $\mathrm{N}$ diets) were performed to assess the variability of the 9 discriminant metabolites of $\mathrm{N}$ diets identified by the untargeted MS method. Data from targeted methods were normalized by creatinine before univariate analyses (2-way ANOVA, $\mathrm{N}$ diets, and sampling techniques with interaction) using XlStat-Biomed (v. 18.1.1, Addinsoft).

\section{RESULTS AND DISCUSSION}

\section{Sampling Techniques Markedly Influenced Urinary Metabolite Profiles}

To compare the number of MS features extracted in each sampling technique, we processed the MS data from TC and SC separately. We obtained similar number of unfiltered features, 3,582 for TC and 3,506 for SC, with $88 \%$ of them common to both sampling techniques (Figure 1). Notwithstanding, when TC and SC data sets were jointly processed to obtain a quantitative comparison of shared features, there were differences between the 2 sampling techniques. After filtration and variable selection, the number of variables was reduced from 4,662 unfiltered features to 2,177 filtered features with more than half $(\mathrm{n}=$ 1,331) of these features being significantly different between sampling techniques (paired parametric test, Benjamini-Hochberg false discovery rate corrected, $P$ $<0.05$; data not shown). Multivariate analyses showed clear separation between metabolite profiles obtained in both sampling techniques (Figure 2). For MS data, separation was observed between individual samples as well as the pooled QC of the 2 sampling techniques. A tight clustering of both QC samples in MS data indicated that the analytical conditions were stable over the time of measurements, and that the HILIC-MS system provided reproducible results. This variation in metabolite profiles could be explained by the difference in the concentration of metabolites between $\mathrm{TC}$ and SC as exemplified in the present study for allantoin and uric acid (Table 1) and also reported by others (Boudra et al., 2012; Lee et al., 2019). Because TC and SC samplings reflected different time spans (i.e., average excretion throughout the day vs. $4 \mathrm{~h}$ postprandial), the proportion of feed and endogenous metabolites may 
Table 1. Concentration of metabolites measured by targeted method (hydrophilic-interaction liquid chromatography-MS/MS) in spot and total urine samples from lactating dairy cows fed 2 dietary nitrogen levels

\begin{tabular}{|c|c|c|c|c|c|c|c|c|}
\hline Item & $\operatorname{Diet}^{1}$ & \multicolumn{7}{|c|}{ Concentration $(\mu \mathrm{mol} / \mathrm{mmol}$ creatinine per $\mathrm{L})$} \\
\hline Total urine collection & LN & $9,106.6$ & $1,010.4$ & $20,734.9^{\mathrm{b}}$ & $1,575.8^{\mathrm{b}}$ & 3.8 & 5.9 & 170.5 \\
\hline \multirow[t]{2}{*}{ Spot urine collection } & $\mathrm{HN}$ & $5,990.6$ & 725.8 & $58,898.3^{\mathrm{a}}$ & $2,024.4^{\mathrm{a}}$ & 4.2 & 7.5 & 476.6 \\
\hline & LN & $7,397.4$ & 786.1 & $22,975.3^{\mathrm{b}}$ & $1,511.0^{\mathrm{b}}$ & 4.0 & 5.0 & 249.6 \\
\hline Diet effect & & 0.113 & 0.987 & $<0.001$ & 0.005 & 0.925 & 0.062 & 0.109 \\
\hline
\end{tabular}

${ }^{\mathrm{a}, \mathrm{b}}$ Mean values within a column and within the same sampling technique with different superscript letters $\operatorname{differ}(P<0.05)$.

${ }^{1} \mathrm{HN}=$ high nitrogen $(14 \% \mathrm{CP}) ; \mathrm{LN}=$ low nitrogen $(11 \% \mathrm{CP})$.

${ }^{2} \beta$ aminobutyric acid.

have differed between both conditions. In addition, it is likely that TC samples also contained modified metabolites induced by sulfuric acid.

\section{Both Sampling Techniques Equally Discriminated Dietary Groups}

Despite the difference in metabolite profiles (Figure 2 ), both sampling techniques were able to discriminate diets in terms of $\mathrm{N}$ content when samples were analyzed by HILIC-ToF/MS and ${ }^{1} \mathrm{H}$ NMR. The PCA models built from MS data showed better separation between diets in terms of $\mathrm{N}$ content than NMR in both sampling techniques (Figure 3). It is also noted that discriminant metabolites were almost the same between sampling techniques (see below) and that the same metabolic pathways were affected by diet independently of the data set analyzed (i.e., SC or TC; MetaboAnalyst pathway analysis, data not shown; http://www.metaboanalyst .ca).

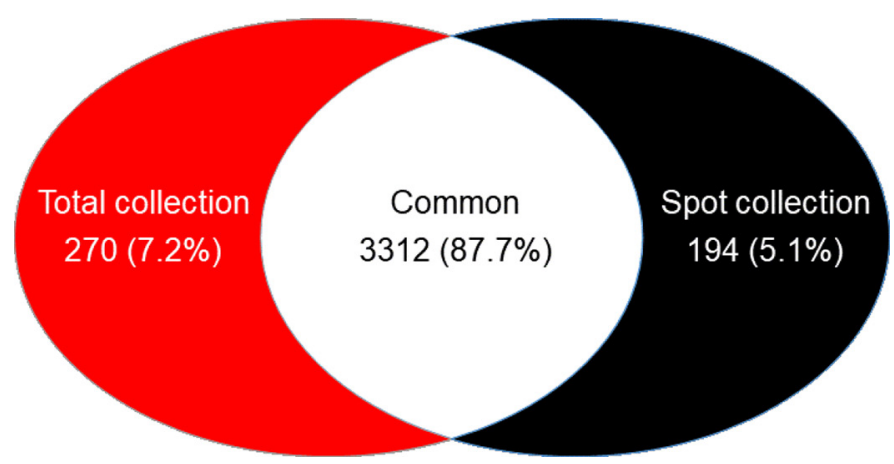

Figure 1. Venn diagram of detected features in untargeted method (hydrophilic-interaction liquid chromatography-time-of-flight-MS) from dairy cow urine collected during $24 \mathrm{~h}$ (total collection) or by spot collection and processed separately.
Several studies reported variability throughout the day in the metabolic profile of urine obtained by $\mathrm{SC}$ compared with the average profile obtained from TC samples (Lee et al., 2019). As stated in the previous section, there were differences due to time spans considered in the collection that were mainly caused by feeding. This difference was also compounded by daily individual differences in feeding behavior, nutrient absorption, and use rate as well as by the time when the animal last urinated. To overcome this drawback, urine outputs from SC were normalized with creatinine as recommended (Shingfield and Offer, 1998; Tebbe and Weiss, 2018). Another normalization method is based on osmolarity (Jacob et al., 2014); whereas other authors recommend repeated sampling throughout 1 or more days (Lee et al., 2019; Wilson et al., 2019). Also, we investigated whether sampling over several days improved the robustness and precision of the SC models. To do that, we compared the quality parameters of PCA and OPLS-DA models of TC built with data obtained in the HILIC-ToF/MS analytical platform from $1 \mathrm{~d}$ (TC-d1) with those built from $1 \mathrm{~d}$ of $\mathrm{SC}$ samples (SC-d1) or the average data of $2,3,4$, or $5 \mathrm{SC}$ samples taken once a day. Both sampling techniques were able to differentiate diets when using PCA models built with data of $d 1$. The separation of $\mathrm{N}$ diet levels was obtained in the first component, whereas separation of energy diets was obtained in the second component. Discrimination of $\mathrm{N}$ diets was achieved with $26.5 \%$ and $20.2 \%$ of variance explained on component 1 for TC-d1 and SC-d1, respectively (Figure 4, Table 2 ). A stronger separation $\left[\mathrm{R}^{2} \mathrm{X}(1)=24 \%\right]$ between $\mathrm{N}$ diet levels was observed when combining data from the 2 first collection days. This value increased marginally when data from more days were averaged, up to $28 \%$ when $5 \mathrm{~d}$ were averaged. The OPLS-DA models showed high value of the predictive parameter $\left(\mathrm{Q}^{2}\right)$ for all the models with a slight increase from 2 -d-average. 

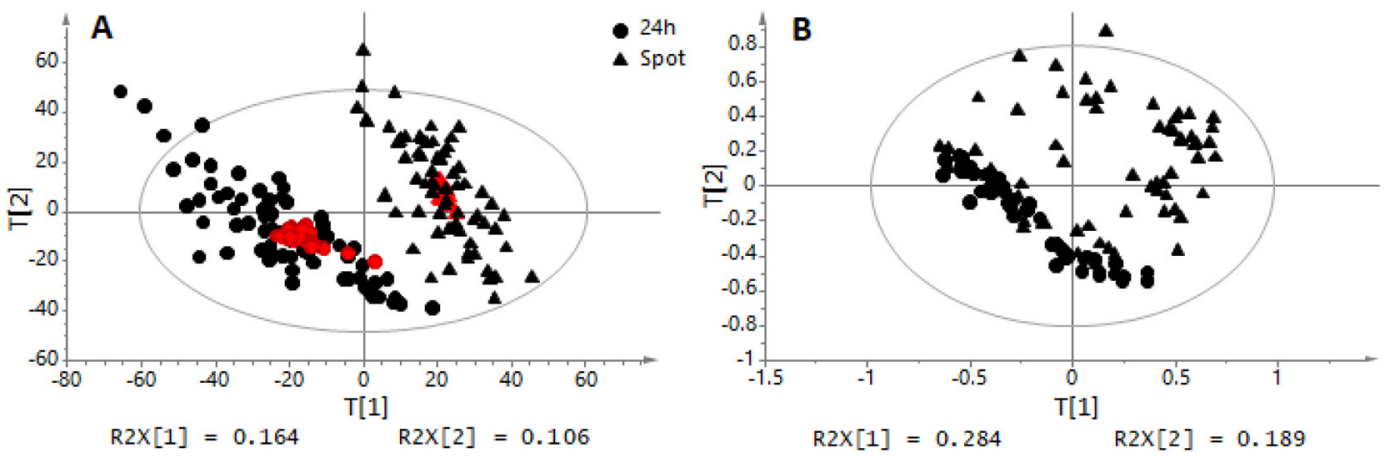

Figure 2. Score plots of principal component analysis of untargeted MS (A) and nuclear magnetic resonance (B) urine metabolites from dairy cows showing grouping of samples depending on the urine collection technique (total $24 \mathrm{~h}$ or spot). Red labels in A designate quality control samples. A tight clustering of quality control samples of both sampling techniques indicates that the analytical conditions were stable over the time of measurements. $\mathrm{R}^{2} \mathrm{X}[1]$ and $\mathrm{R}^{2} \mathrm{X}[2]$ represent the explained variance in component 1 (T1) and 2 (T2), respectively.

Although good discrimination is obtained at $\mathrm{d} 1$ with a $\mathrm{Q}^{2}$ value similar to that of $\mathrm{TC}(\mathrm{SC}-1 \mathrm{~d}=0.81 ; \mathrm{TC}-1 \mathrm{~d}=$ $0.73)$, we observed a better discriminative power from 2-d-average $(\mathrm{SC}-2 \mathrm{~d}=0.89)$ that remained stable over $5 \mathrm{~d}$ of collection (Table 2). The number of VIP $\geq 1$, indicating the contribution of variables to the discrimination between $\mathrm{N}$ diets, was similar among models, with the exception of model built with data from $\mathrm{d}$ 1 that is smaller. These observations suggested that multiple-day collection improved the model quality, and that 2 consecutive days seemed enough to achieve stable results. This hypothesis is consistent with the Venn diagram (Supplemental Figure S1, https://data .inrae.fr/dataset.xhtml?persistentId=doi:10.15454/ FFFRL2, Morgavi, 2021) obtained by comparing VIP lists extracted from each OPLS-DA model (VIP $\geq 1$ ). Indeed, when looking at the proportion of variables specific to each model, we observed that it was higher in the SC-1-d model and decreased with the successive addition of collection days except for SC averaged over
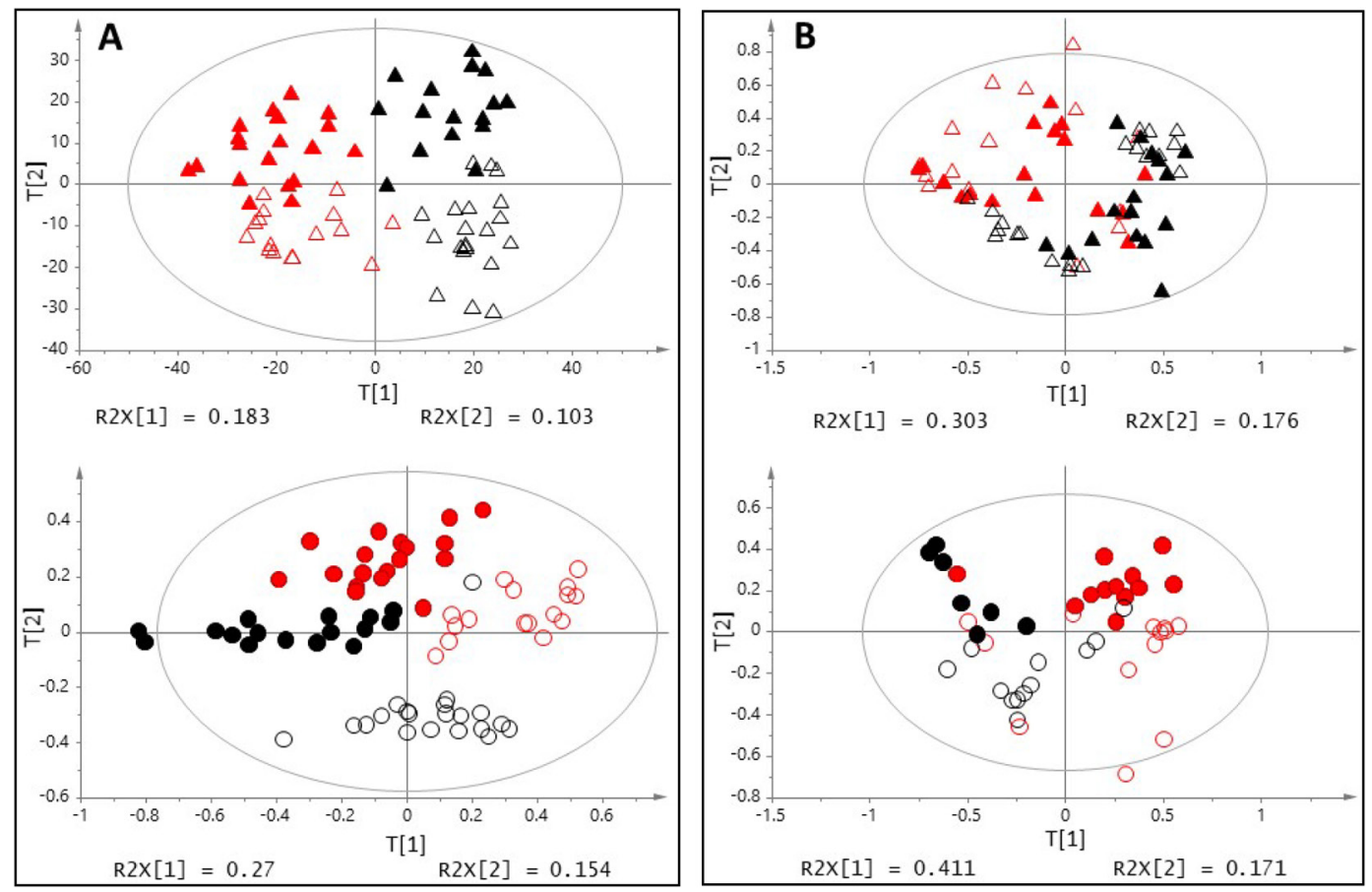

Figure 3. Scores plot of principal component analysis of untargeted MS (A) and nuclear magnetic resonance (B) urine metabolites from total (circle) and spot (triangle) collections from dairy cows fed the following 4 diets: high (red labels) or low (black labels) in nitrogen with starch (full label) or fiber (empty label) as energy source. Samples were collected over $5 \mathrm{~d}$. $\mathrm{R}^{2} \mathrm{X}[1]$ and $\mathrm{R}^{2} \mathrm{X}[2]$ represent the explained variance in component 1 (T1) and 2 (T2), respectively. 

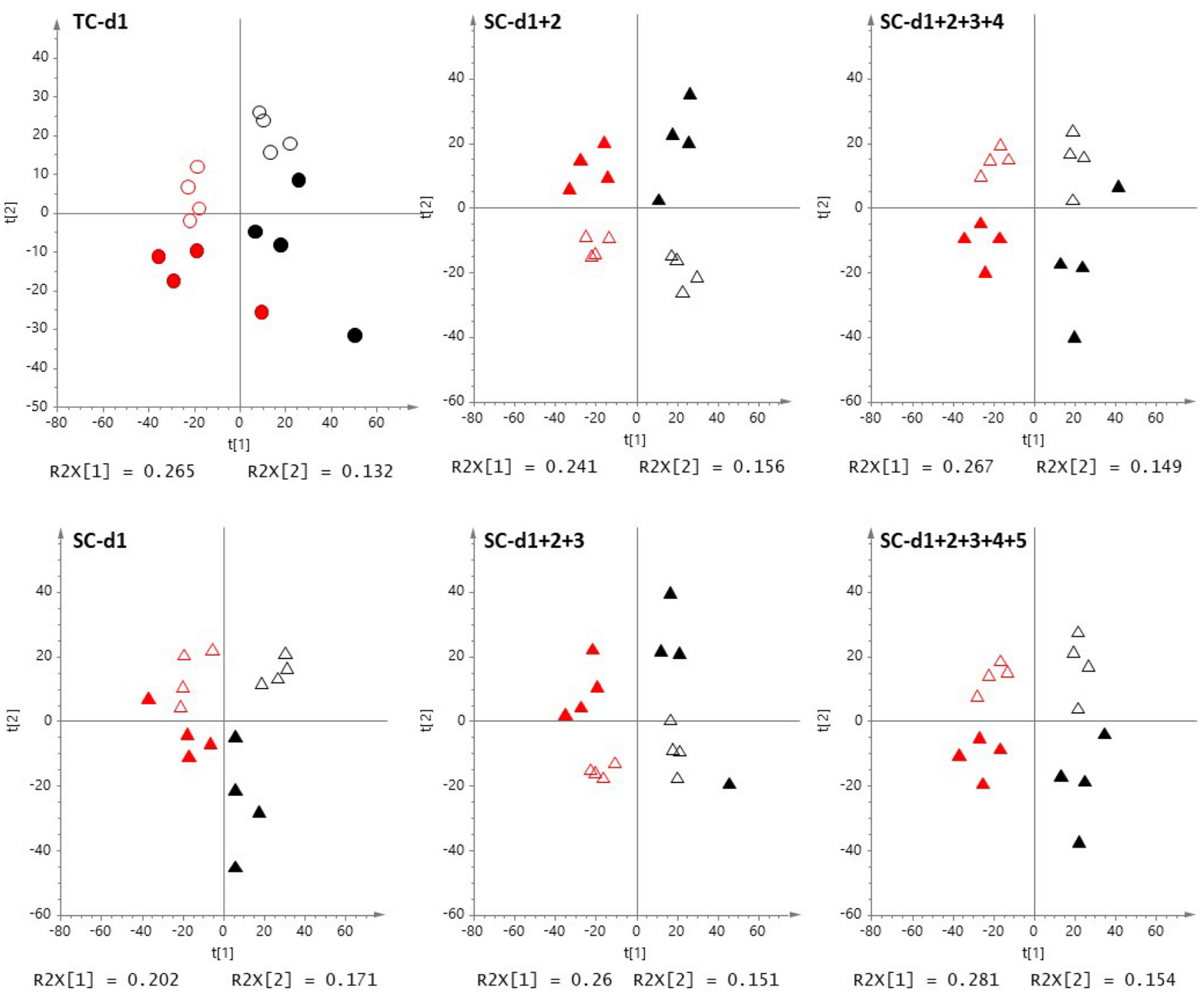

Figure 4. Comparison of principal component analysis score plots of hydrophilic-interaction liquid chromatography-time-of-flight-MS urine data obtained from total collection at $1 \mathrm{~d}$ (TC-d1, circle) versus spot collection (triangle) built with data from $1 \mathrm{~d}$ (SC-d1) and the mean data of $2,3,4$, and $5 \mathrm{~d}(\mathrm{SC}-\mathrm{d} 1+2$ to $\mathrm{d} 1+2+3+4+5)$. Urine samples were obtained from dairy cows fed diets high (red labels) or low (black labels) in nitrogen with starch (full labels) and fiber (empty labels) as energy source. Nitrogen level in the diet was discriminant in the first component, whereas starch level was discriminant in the second component. $\mathrm{R}^{2} \mathrm{X}[1]$ and $\mathrm{R}^{2} \mathrm{X}[2]$ represent the explained variance in component 1 (T1) and 2 (T2), respectively.

5 d. Again, these results agree with the suggestion that collection during $2 \mathrm{~d}$ (2-d average) would be necessary and potentially sufficient.

To highlight discriminant metabolites associated with differences in diets in terms of $\mathrm{N}$ content, OPLS-DA models were built from samples collected over $5 \mathrm{~d}$ from both sampling techniques. We focused on the possible differences between high and low $\mathrm{CP}$ diets because of the importance that dietary $\mathrm{N}$ content has on animal production and N pollution (Castillo et al., 2000). However, because the nature of the energy supply also had a significant effect on the urine metabolome as showed in Figure 4, the OPLS models were performed separately for each energy source (starch and fiber). Within each energy source, a clear separation of diets differing in $\mathrm{N}$ content was obtained with both sampling techniques with a good predictive capacity $\left(\mathrm{Q}^{2}\right)$ for $\mathrm{MS}$ and NMR data (Supplemental Figure S2, https://data.inrae.fr/ dataset.xhtml?persistentId=doi:10.15454/FFFRL2, Morgavi, 2021). Twenty metabolites discriminating N diets were identified as follows: 11 by NMR (Supplemental Table S2, https://data.inrae.fr/dataset.xhtml 
?persistentId=doi:10.15454/FFFRL2, Morgavi, 2021) and confirmed by NMR 2-D in urine spiked with pure standards (Supplemental Figure S3, https://data.inrae .fr/dataset.xhtml?persistentId=doi:10.15454/FFFRL2, Morgavi, 2021), and 9 by HILIC-ToF/MS (Supplemental Table S3, https://data.inrae.fr/dataset.xhtml ?persistentId=doi:10.15454/FFFRL2, Morgavi, 2021). These discriminant metabolites were common to both sampling techniques, and followed the same pattern, (i.e., increase or decrease). Notwithstanding, in agreement with Lee et al. (2019), higher variability between cows was observed for some metabolites detected by MS in SC compared with TC samples (Supplemental Figure S4, https://data.inrae.fr/dataset.xhtml?persistentId= doi:10.15454/FFFRL2, Morgavi, 2021).

We also compared both sampling techniques using a quantitative HILIC-MS/MS method targeting 9 markers of nitrogen status as follows: allantoin, uric acid, xanthine, hypoxanthine, $\beta$-aminobutyric acid, $\beta$-alanine, creatinine, creatine, and urea (Boudra et al., 2012). Results showed no difference $(P>0.05)$ of the sampling technique in the concentration of these metabolites, except for allantoin and uric acid that were significantly $(P<0.05)$ higher in TC (Figure $5)$. These differences could be explained in particular by diurnal variation in purine derivative urinary excretion (Tas and Susenbeth, 2007). When considering $\mathrm{N}$ level, all metabolites followed similar patterns in both sampling techniques, and creatine and urea were significantly different $(P<0.05)$ between $\mathrm{N}$ diets (Table 1). Moreover, the interaction (diet $\times$ sampling technique) in our study was not significant, indicat- ing that detection of the diet effect was similar for both sampling techniques. Other studies reported no differences between TC and SC for estimating daily purine derivatives excretion in ruminants when urinary output was estimated from creatinine concentration in SC samples (Valadares et al., 1999; Chizzotti et al., 2008). Nonetheless, urine creatinine concentration does vary throughout the day (Lee et al., 2019) and should be considered depending on the objective of the study as discussed below. Similar to untargeted results, quantification of all metabolites in individual cows followed similar pattern in both collections (Supplemental Figure S5, https://data.inrae.fr/dataset .xhtml?persistentId=doi:10.15454/FFFRL2, Morgavi, 2021). Supporting these results, linear regression analyses showed a strong correlation between sampling techniques for metabolites analyzed by the targeted method (Boudra et al., 2012). Human clinical studies showed that single urine SC samples collected in the morning and analyzed by the untargeted NMR method allowed the discrimination between healthy and sick groups (Saude et al., 2011). Alternatively, Wilson et al. (2019) showed that there was no significant loss of information when TC was substituted for SC in human studies. To our knowledge, no such studies were performed in ruminants. It is acknowledged, however, that metabolomics studies require a high number of samples, and these results should be validated using larger groups of animals. Also, a single urine SC as used in this work cannot capture variations throughout the day or be used for the 24-h quantitative estimation of metabolites excretion.

Table 2. Comparative ability of total and spot urine collection methods to detect differences in the metabolome obtained by untargeted method (hydrophilic-interaction liquid chromatography-time-of-flight-MS) of lactating dairy cows induced by diet

\begin{tabular}{|c|c|c|c|c|c|c|c|}
\hline \multirow[b]{4}{*}{ Data $^{1}$} & \multicolumn{7}{|c|}{ Quality of multivariate model } \\
\hline & \multicolumn{3}{|c|}{$\mathrm{PCA}^{2}$} & \multicolumn{4}{|c|}{ OPLS-DA $^{3}$} \\
\hline & \multicolumn{3}{|c|}{ Discrimination on $\mathrm{C} 1$} & \multicolumn{4}{|c|}{ Model performance } \\
\hline & All diets ${ }^{4}$ & $\mathrm{~N}$ diets & $\mathrm{R}^{2}(\mathrm{X})$ & $\mathrm{PC}$ & $\mathrm{R}^{2}(\mathrm{X})$ & $\mathrm{Q}^{2}$ & VIP \\
\hline $\mathrm{TC} 1 \mathrm{~d}$ & Partial & Partial & 26.5 & $1+0+0$ & 0.20 & 0.73 & \\
\hline $\mathrm{SC} 1 \mathrm{~d}$ & Partial & Partial & 20.2 & $1+1+0$ & 0.29 & 0.81 & 0.05 \\
\hline SC 2-d mean & Yes & Yes & 24.1 & $1+1+0$ & 0.29 & 0.89 & 0.03 \\
\hline SC 3-d mean & Yes & Yes & 26.0 & $1+0+0$ & 0.26 & 0.88 & 0.01 \\
\hline SC 4-d mean & Yes & Yes & 26.7 & $1+1+0$ & 0.36 & 0.88 & 0.01 \\
\hline SC 5-d mean & Yes & Yes & 28.1 & $1+0+0$ & 0.28 & 0.89 & 0.03 \\
\hline
\end{tabular}

${ }^{1}$ Mass spectrometry data obtained from total (TC) and spot collection (SC) samples obtained from one day $(1 \mathrm{~d})$ or averaged over 2 to 5 consecutive days of sampling for $\mathrm{SC} \cdot{ }^{2} \mathrm{PCA}=$ principal component analysis; $\mathrm{C} 1$ $=$ component 1 .

${ }^{3}$ OPLS-DA = orthogonal partial least squares-discriminant analysis $; \mathrm{PC}=$ principal component; $\mathrm{R}^{2}(\mathrm{X})=$ variance explained by the 2 components; $\mathrm{Q}^{2}=$ predictive parameter of the model; VIP $=$ ratio of specific variable importance in projection (VIP)/total VIP in the model.

${ }^{4}$ Four diets containing $14 \%$ or $11 \% \mathrm{CP}$ with starch or fiber as energy source. 

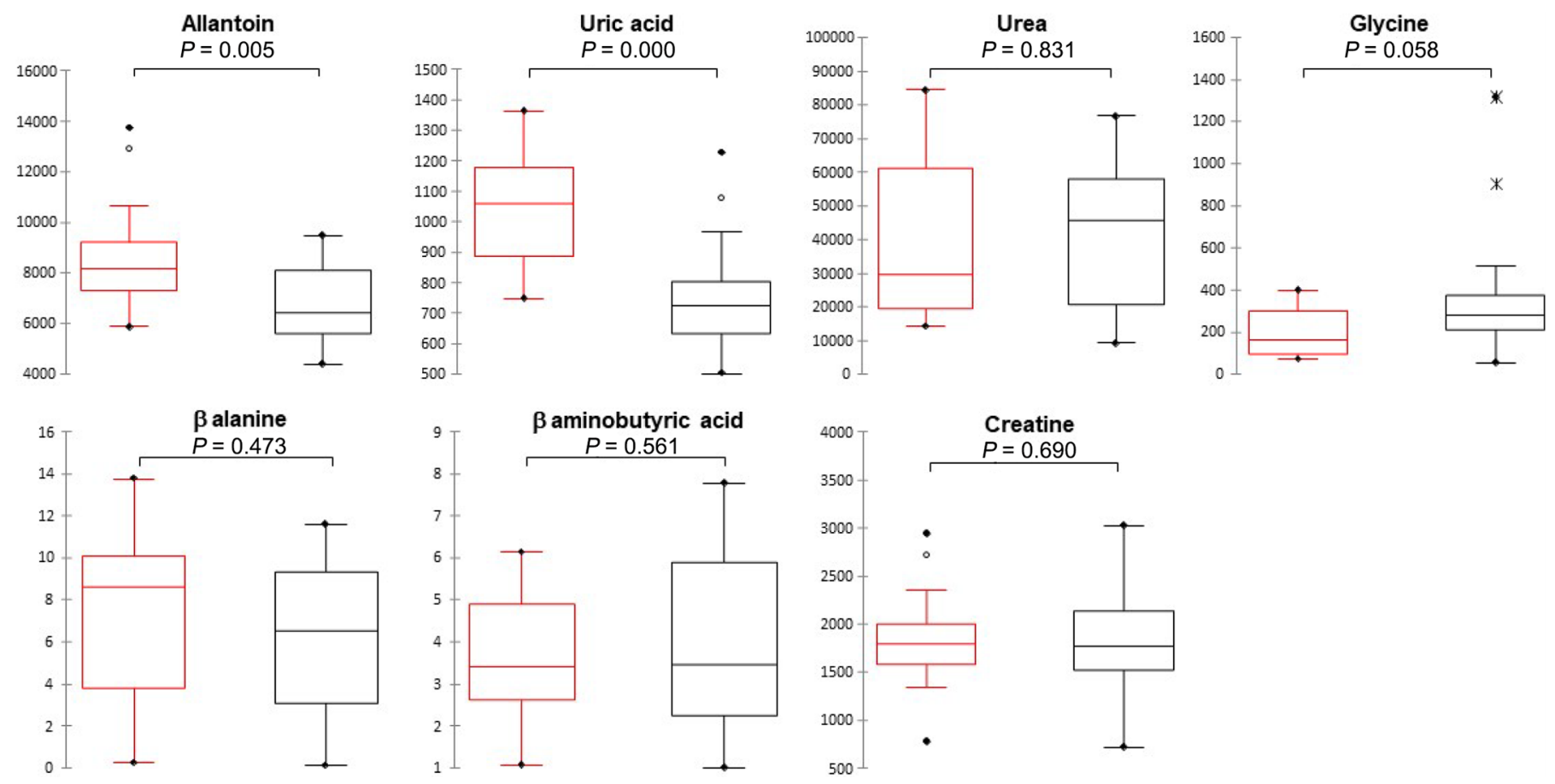

Figure 5. The y-axis represents the concentration of 7 urine metabolites quantified by hydrophilic-interaction liquid chromatography-tandem MS from total (red) and spot (black) urine samples from lactating dairy cows. The concentration of allantoin and uric acid differed significantly $(P<0.05)$ between sampling collection technique. For each box, the central horizontal bar is the median; the lower and upper limits of the box are the first and third quartiles, respectively; and points above or below the maximum and minimum value may be considered as outliers.

A total of 22 metabolites discriminating the dietary $\mathrm{N}$ level were identified from the 3 analytical platforms as follows: 20 metabolites from untargeted methods (Supplemental Tables S2 and S3) and 2 metabolites from the targeted method (Table 1). Three common metabolites were found in at least 2 analytical platforms (p-cresol sulfate, phenyl-acetylglycine, and urea). $\mathrm{P}$-cresol is a rumen metabolite of tyrosine, and approximately half of the tyrosine content of rumen-administered casein was excreted as p-cresol in sheep (Martin, 1982). Interestingly, p-cresol and phenyl-acetylglycine are metabolically related. Indeed, the aromatic AA, phenylalanine and tyrosine, are first converted into phenyl acetate and p-cresol through the action of the gut microbiota (Smith and Macfarlane, 1996) and then conjugated with glycine and glucuronide, respectively, to form phenyl-acetylglycine and p-cresol glucuronide in the liver and gut mucosa (De Pascali et al., 2017). This suggests that urinary p-cresol and phenyl-acetylglycine concentration may reflect the intake of dietary phenylalanine and tyrosine in ruminants, and thus be a proxy of the overall $\mathrm{N}$ intake. Concerning the urinary urea concentration as a biomarker of $\mathrm{N}$ intake, it is well established that the proportion of urea in urine increases as the $\mathrm{N}$ intake increases (Gonda and Lindberg, 1994), and urea concentration in blood or milk have been proposed as a biomarker of the N-use efficiency and $\mathrm{N}$ intake in ruminants (Kohn et al., 2005; Huhtanen et al., 2015).

Further regression models based on these 22 discriminant metabolites were constructed to investigate the relationships between these metabolites and the $\mathrm{N}$-use efficiency at the digestive, metabolic, and rumen levels. There was no relationship except for the overall milk N-use efficiency (Supplemental Figure S6, ttps://data .inrae.fr/dataset.xhtml?persistentId=doi:10.15454/ FFFRL2, Morgavi, 2021), likely due to the inherent errors associated with digestive and metabolic measurements compared with the milk N-use efficiency.

This work showed that SC is as good as TC for obtaining robust metabolomics results in lactating cows. When applied to assess the effect of dietary $\mathrm{N}$ level, urine samples obtained from SC effectively discriminated diets with high or low N levels. Spot urine collection also avoids the practical limitations and animal welfare issues involved in TC, making it highly recommendable for metabolomics studies in lactating cows.

\section{ACKNOWLEDGMENTS}

This work was funded by the seventh EU framework project "Innovative and practical management ap- 
proaches to reduce nitrogen excretion by ruminants (REDNEX)," FP7, KBB-2007-1. We thank David Alvarez and Ulli Hohenester (UMRH, INRAE) for their technical assistance. We also thank the personnel of the experimental animal facilities for the care of animals. The authors have not stated any conflicts of interest.

\section{REFERENCES}

Beckonert, O., H. C. Keun, T. M. D. Ebbels, J. Bundy, E. Holmes, J. C. Lindon, and J. K. Nicholson. 2007. Metabolic profiling, metabolomic and metabonomic procedures for NMR spectroscopy of urine, plasma, serum and tissue extracts. Nat. Protoc. 2:26922703. https://doi.org/10.1038/nprot.2007.376.

Bertram, H. C., C. Yde, X. Zhang, and N. Kristensen. 2011. Effect of dietary nitrogen content on the urine metabolite profile of dairy cows assessed by nuclear magnetic resonance (NMR)-based metabolomics. J. Agric. Food Chem. 59:12499-12505. https://doi.org/10 $.1021 /$ jf204201f

Boomsma, F., G. Alberts, L. van Eijk, A. J. Man in 't Veld, and M. A. Schalekamp. 1993. Optimal collection and storage conditions for catecholamine measurements in human plasma and urine. Clin. Chem. 39:2503-2508. https://doi.org/10.1093/clinchem/39 .12 .2503 .

Boudra, H., M. Doreau, P. Noziere, E. Pujos-Guillot, and D. P. Morgavi. 2012. Simultaneous analysis of the main markers of nitrogen status in dairy cow's urine using hydrophilic interaction chromatography and tandem mass spectrometry detection. J. Chromatogr. A 1256:169-176. https://doi.org/10.1016/j.chroma.2012.07.094.

Cantalapiedra-Hijar, G., R. J. Dewhurst, L. Cheng, A. R. J. Cabrita, A. J. M. Fonseca, P. Noziere, D. Makowski, H. Fouillet, and I. Ortigues-Marty. 2018. Nitrogen isotopic fractionation as a biomarker for nitrogen use efficiency in ruminants: A meta-analysis. Animal 12:1827-1837. https://doi.org/10.1017/S1751731117003391.

Castillo, A. R., E. Kebreab, D. E. Beever, and J. France. 2000. A review of efficiency of nitrogen utilisation in lactating dairy cows and its relationship with environmental pollution. J. Anim. Feed Sci. 9:1-32. https://doi.org/10.22358/jafs/68025/2000.

Chen, X. B., and M. J. Gomes. 1992. Estimation of microbial protein supply to sheep and cattle based on urinary excretion of purine derivatives-An overview of the technical details. Int. Feed Resources Unit, Rowett Res. Inst., Occasional Publ.

Chizzotti, M. L., S. C. Valadares Filho, R. F. D. Valadares, F. H. M. Chizzotti, and L. O. Tedeschi. 2008. Determination of creatinine excretion and evaluation of spot urine sampling in Holstein cattle. Livest. Sci. 113:218-225. https://doi.org/10.1016/j.livsci.2007.03 .013 .

Chowdhury, S. K., V. Katta, R. C. Beavis, and B. T. Chait. 1990. Origin and removal of adducts (molecular mass $=98 \mathrm{u}$ ) attached to peptide and protein ions in electrospray ionization mass spectra. J. Am. Soc. Mass Spectrom. 1:382-388. https://doi.org/10.1016/ 1044-0305(90)85018-H.

Contreras-Jodar, A., N. H. Nayan, S. Hamzaoui, G. Caja, and A. A. K. Salama. 2019. Heat stress modifies the lactational performances and the urinary metabolomic profile related to gastrointestinal microbiota of dairy goats. PLoS One 14:e0202457. https://doi.org/10 .1371/journal.pone.0202457.

De Pascali, S. A., L. Gambacorta, I. P. Oswald, L. Del Coco, M. Solfrizzo, and F. P. Fanizzi. 2017. (1)H NMR and MVA metabolomic profiles of urines from piglets fed with boluses contaminated with a mixture of five mycotoxins. Biochem. Biophys. Rep. 11:9-18. https://doi.org/10.1016/j.bbrep.2017.05.004.

Dunn, W. B., D. I. Broadhurst, H. J. Atherton, R. Goodacre, and J. L. Griffin. 2011. Systems level studies of mammalian metabolomes: The roles of mass spectrometry and nuclear magnetic resonance spectroscopy. Chem. Soc. Rev. 40:387-426. https://doi.org/10 $.1039 /$ B906712B.

Fan, T. W. M. 1996. Metabolite profiling by one- and two-dimensional NMR analysis of complex mixtures. Prog. Nucl. Magn.
Reson. Spectrosc. 28:161-219. https://doi.org/10.1016/0079 -6565(95)01017-3.

Fanchone, A., P. Noziere, J. Portelli, B. Duriot, V. Largeau, and M. Doreau. 2013. Effects of nitrogen underfeeding and energy source on nitrogen ruminal metabolism, digestion, and nitrogen partitioning in dairy cows. J. Anim. Sci. 91:895-906. https://doi.org/10 $.2527 /$ jas.2012-5296.

Fernández-Peralbo, M. A., and M. D. Luque de Castro. 2012. Preparation of urine samples prior to targeted or untargeted metabolomics mass-spectrometry analysis. Trends Analyt. Chem. 41:75-85. https://doi.org/10.1016/j.trac.2012.08.011.

Furey, A., M. Moriarty, V. Bane, B. Kinsella, and M. Lehane. 2013. Ion suppression; A critical review on causes, evaluation, prevention and applications. Talanta 115:104-122. https://doi.org/10.1016/j .talanta.2013.03.048.

Giacomoni, F., G. Le Corguille, M. Monsoor, M. Landi, P. Pericard, M. Petera, C. Duperier, M. Tremblay-Franco, J. F. Martin, D. Jacob, S. Goulitquer, E. A. Thevenot, and C. Caron. 2015. Workflow4Metabolomics: A collaborative research infrastructure for computational metabolomics. Bioinformatics 31:1493-1495. https: //doi.org/10.1093/bioinformatics/btu813.

Gonda, H. L., and J. E. Lindberg. 1994. Evaluation of dietary nitrogen utilization in dairy cows based on urea concentrations in blood, urine and milk, and on urinary concentration of purine derivatives. Acta Agric. Scand. A Anim. Sci. 44:236-245. https://doi.org/10 $.1080 / 09064709409410904$.

Harris, C. I., and G. Milne. 1981. The urinary excretion of $\mathrm{N}$ taumethyl histidine by cattle: Validation as an index of muscle protein breakdown. Br. J. Nutr. 45:411-422. https://doi.org/10.1079/ BJN19810116.

Huhtanen, P., E. Cabezas-Garcia, S. Krizsan, and K. Shingfield. 2015. Evaluation of between-cow variation in milk urea and rumen ammonia nitrogen concentrations and the association with nitrogen utilization and diet digestibility in lactating cows. J. Dairy Sci. 98:3182-3196. https://doi.org/10.3168/jds.2014-8215.

Jacob, C. C., G. Dervilly-Pinel, G. Biancotto, and B. Le Bizec. 2014 Evaluation of specific gravity as normalization strategy for cattle urinary metabolome analysis. Metabolomics 10:627-637. https:// doi.org/10.1007/s11306-013-0604-z.

Kim, K. M., G. N. Henderson, R. F. Frye, C. D. Galloway, N. J. Brown, M. S. Segal, W. Imaram, A. Angerhofer, and R. J. Johnson. 2009. Simultaneous determination of uric acid metabolites allantoin, 6-aminouracil, and triuret in human urine using liquid chromatography-mass spectrometry. J. Chromatogr. B Analyt. Technol. Biomed. Life Sci. 877:65-70. https://doi.org/10.1016/j .jchromb.2008.11.029.

Kirwan, J. A., L. Brennan, D. Broadhurst, O. Fiehn, M. Cascante, W B. Dunn, M. A. Schmidt, and V. Velagapudi. 2018. Preanalytical processing and biobanking procedures of biological samples for metabolomics research: A white paper, community perspective (for "precision medicine and pharmacometabolomics task group"-the metabolomics society initiative). Clin. Chem. 64:1158-1182. https: //doi.org/10.1373/clinchem.2018.287045.

Kohn, R. A., M. M. Dinneen, and E. Russek-Cohen. 2005. Using blood urea nitrogen to predict nitrogen excretion and efficiency of nitrogen utilization in cattle, sheep, goats, horses, pigs, and rats. J. Anim. Sci. 83:879-889. https://doi.org/10.2527/2005.834879x.

Lee, C., D. L. Morris, and P. A. Dieter. 2019. Validating and optimizing spot sampling of urine to estimate urine output with creatinine as a marker in dairy cows. J. Dairy Sci. 102:236-245. https://doi .org/10.3168/jds.2018-15121.

Martin, A. K. 1982. The origin of urinary aromatic compounds excreted by ruminants. 3 . The metabolism of phenolic compounds to simple phenols. Br. J. Nutr. 48:497-507. https://doi.org/10.1079/ BJN19820135.

Morgavi, D. 2021. Supporting data for "Spot urine collection: A valid alternative to total urine collection for metabolomic studies in dairy cattle." https://doi.org/10.15454/FFFRL2, Portail Data INRAE, V1.

Nalon, E., and P. Stevenson. 2019. Protection of dairy cattle in the EU: State of play and directions for policymaking from a legal and 
animal advocacy perspective. Animals (Basel) 9:1066. https://doi .org/10.3390/ani9121066.

Patra, A. K., and J. R. Aschenbach. 2018. Ureases in the gastrointestinal tracts of ruminant and monogastric animals and their implication in urea-N/ammonia metabolism: A review. J. Adv. Res. 13:39-50. https://doi.org/10.1016/j.jare.2018.02.005.

Saude, E. J., C. D. Skappak, S. Regush, K. Cook, A. Ben-Zvi, A. Becker, R. Moqbel, B. D. Sykes, B. H. Rowe, and D. J. Adamko. 2011. Metabolomic profiling of asthma: Diagnostic utility of urine nuclear magnetic resonance spectroscopy. J. Allergy Clin. Immunol. 127:757-764.E6. https://doi.org/10.1016/j.jaci.2010.12.1077.

Schrimpe-Rutledge, A. C., S. G. Codreanu, S. D. Sherrod, and J. A. McLean. 2016. Untargeted metabolomics strategies - Challenges and emerging directions. J. Am. Soc. Mass Spectrom. 27:18971905. https://doi.org/10.1007/s13361-016-1469-y.

Shingfield, K. J., and N. W. Offer. 1998. Evaluation of the spot urine sampling technique to assess urinary purine derivative excretion in lactating dairy cows. Anim. Sci. 66:557-568. https://doi.org/10 $.1017 / \mathrm{S} 1357729800009139$.

Smith, E. A., and G. T. Macfarlane. 1996. Enumeration of human colonic bacteria producing phenolic and indolic compounds: Effects of $\mathrm{pH}$, carbohydrate availability and retention time on dissimilatory aromatic amino acid metabolism. J. Appl. Bacteriol. 81:288-302. https://doi.org/10.1111/j.1365-2672.1996.tb04331.x.

Sun, H. Z., D. M. Wang, B. Wang, J. K. Wang, H. Y. Liu, L. L. Guan, and J.-X. Liu. 2015. Metabolomics of four biofluids from dairy cows: potential biomarkers for milk production and quality. J. Proteome Res. 14:1287-1298. https://doi.org/10.1021/pr501305g.

Tas, B. M., and A. Susenbeth. 2007. Urinary purine derivates excretion as an indicator of in vivo microbial $\mathrm{N}$ flow in cattle: A review. Livest. Sci. 111:181-192. https://doi.org/10.1016/j.livsci.2007.05 .010 .

Tebbe, A. W., and W. P. Weiss. 2018. Evaluation of creatinine as a urine marker and factors affecting urinary excretion of magnesium by dairy cows. J. Dairy Sci. 101:5020-5032. https://doi.org/ 10.3168/jds.2017-14098.

Ulrich, E. L., H. Akutsu, J. F. Doreleijers, Y. Harano, Y. E. Ioannidis, J. Lin, M. Livny, S. Mading, D. Maziuk, Z. Miller, E. Nakatani, C. F. Schulte, D. E. Tolmie, R. Kent Wenger, H. Yao, and J. L. Markley. 2008. BioMagResBank. Nucleic Acids Res. 36(Database):D402-D408. https://doi.org/10.1093/nar/gkm957.

Valadares, R. F. D., G. A. Broderick, S. C. V. Filho, and M. K. Clayton. 1999. Effect of replacing alfalfa silage with high moisture corn on ruminal protein synthesis estimated from excretion of total purine derivatives. J. Dairy Sci. 82:2686-2696. https://doi.org/10 .3168/jds.S0022-0302(99)75525-6.

van der Kloet, F. M., I. Bobeldijk, E. R. Verheij, and R. H. Jellema. 2009. Analytical error reduction using single point calibration for accurate and precise metabolomic phenotyping. J. Proteome Res. 8:5132-5141. https://doi.org/10.1021/pr900499r.

Vuckovic, D. 2012. Current trends and challenges in sample preparation for global metabolomics using liquid chromatography-mass spectrometry. Anal. Bioanal. Chem. 403:1523-1548. https://doi .org/10.1007/s00216-012-6039-y.

Wilson, T., I. Garcia-Perez, J. M. Posma, A. J. Lloyd, E. S. Chambers, K. Tailliart, H. Zubair, M. Beckmann, J. C. Mathers, E. Holmes, G. Frost, and J. Draper. 2019. Spot and cumulative urine samples are suitable replacements for 24-hour urine collections for objective measures of dietary exposure in adults using metabolite biomarkers. J. Nutr. 149:1692-1700. https://doi.org/10.1093/jn/ nxz138.

Wishart, D. S., Y. D. Feunang, A. Marcu, A. C. Guo, K. Liang, R. Vazquez-Fresno, T. Sajed, D. Johnson, C. Li, N. Karu, Z. Sayeeda, E. Lo, N. Assempour, M. Berjanskii, S. Singhal, D. Arndt, Y. Liang, H. Badran, J. Grant, A. Serra-Cayuela, Y. Liu, R. Mandal, V. Neveu, A. Pon, C. Knox, M. Wilson, C. Manach, and A. Scalbert. 2018. HMDB 4.0: the human metabolome database for 2018 . Nucleic Acids Res. 46(D1):D608-D617. https://doi.org/10.1093/ nar/gkx1089.

Worley, B., and R. Powers. 2013. Multivariate analysis in metabolomics. Curr. Metabolomics 1:92-107. https://doi.org/10.2174/ $2213235 X 11301010092$.

Worley, B., and R. Powers. 2016. PCA as a practical indicator of OPLS-DA model reliability. Curr. Metabolomics 4:97-103. https: /doi.org/10.2174/2213235X04666160613122429.

\section{ORCIDS}

P. Noziere $\odot$ https://orcid.org/0000-0003-1727-8984

G. Cantalapiedra-Hijar @ https://orcid.org/0000-0001-9486-8238

M. Petera @ https://orcid.org/0000-0002-1779-6901

D. P. Morgavi $\odot$ https://orcid.org/0000-0002-3883-0937 